\title{
A generalization of inverse distance weighting method via kernel regression and its application to surface modeling
}

\author{
Chuanfa Chen $\cdot$ Na Zhao $\cdot$ Tianxiang Yue $\cdot$ Jinyun Guo
}

Received: 3 July 2014 / Accepted: 11 November 2014 / Published online: 26 November 2014

(C) Saudi Society for Geosciences 2014

\begin{abstract}
The inverse-distance weighting (IDW) method is considered as one of the most popular deterministic methods and is widely applied to a variety of areas because of its low computational cost and easy implementation. In this paper, we show that the classical IDW is essentially a zeroth-order local kernel regression method with an inverse distance weight function. Thus, it suffers from various shortcomings, such as the boundary bias. Considering the advantages of the local polynomial modeling technique in statistics, the classical IDW was generalized into a higher-order regression by the Taylor expansion and then computed by means of a weighted leastsquares method. Surface modeling of rainfall fields in China indicated that the generalized IDWs with the first- and secondorders are more accurate than the classical IDW in terms of root mean square error (RMSE). The example of digital elevation model construction with a group of sample points showed that the two generalized IDWs have better RMSE and mean error than the classical IDW. Furthermore, the second-order IDW has a better performance than the ordinary kriging in terms of RMSE. A theoretical analysis demonstrated that the gradient-plus-inverse distance squared method presented by Nalder and Wein (Agric For Meteorol 92(4):
\end{abstract}

C. Chen $(\bowtie) \cdot$ J. Guo

State Key Laboratory of Mining Disaster Prevention and Control Co-founded by Shandong Province and the Ministry of Science and Technology, Shandong University of Science and Technology, 266590 Qingdao, China

e-mail: chencf@1reis.ac.cn

C. Chen $\cdot$ J. Guo

Geomatics College, Shandong University of Science and

Technology, 266590 Qingdao, China

N. Zhao $\cdot$ T. Yue

State Key Laboratory of Resources and Environment Information System, Institute of Geographical Sciences and Natural Resources Research, 100101 Beijing, China
211-225, 1998) is a first-order form of the generalized IDW expanded on spatial coordinates and elevation. In a word, the generalized IDW can incorporate multiple covariates, which can better explain the interpolation procedure and might improve its accuracy.

Keywords IDW · Interpolation · Surface modeling · DEM · Rainfall

\section{Introduction}

Spatial interpolation is a procedure of estimating the value of an attribute at a location by using values of the same attribute sampled at neighbor points (Chen and Yue 2010; Falivene et al. 2010; Lloyd 2005). The guiding principle for all spatial interpolation methods is the Tobler's first law of geography, which states that everything is related to everything else, but nearby things are more related than distant things. Interpolation is mainly used to convert data from point observations to continuous fields so that the spatial patterns sampled by these measurements can be compared with the spatial patterns of other entities (Burrough and McDonnell 1998). Spatially interpolated continuous fields are widely used as inputs to a broad variety of modeling activities. These include ecological, geomorphological, epidemiological, and hydrological modeling procedures (Moore et al. 1991).

Broadly, interpolation methods can be defined as being probabilistic and deterministic (Delbari 2014; Khashei-Siuki and Sarbazi 2013; Shahbeik et al. 2014). Both the two types of methods can be applied using all the available data (global neighborhood) or using a subset of nearby data within a local neighborhood centered on the location where interpolation is performed. Probabilistic geostatistical methods rely on spatial autocorrelation, and account for distance and direction when determining the importance of points used for interpolation 
(Oliver and Webster 1990) whereas, in deterministic methods, the point influence is modeled based on assumptions, but empirically derived. The inverse-distance weighting (IDW) method is one of the most popular deterministic methods because of its low computational cost and easy implementation. Moreover, in various practical applications, a large number of comparative studies between different interpolators indicated that IDW may be as good as or better than krigingbased techniques when there is a problem of making meaningful estimates of the field spatial structure from sparse data (Boman et al. 1995; Brus et al. 1996; Gallichand and Marcotte 1993; Kravchenko 2003; Weber and Englund 1992). At present, there has been a large body of literature on applications of IDW into a variety of fields, and it may be concluded that IDW is being established as a necessary interpolation tool in many GIS software packages.

Despite its popularity, the classical IDW always suffers from several limitations such as the constant and isotropy distance-decay relationships throughout the entire study area. Thus, many improved versions of IDW have been developed. Some researchers tried to improve the ability of the classical IDW to deal with anisotropic observations. For example, Tomczak (1998) developed an automated anisotropic IDW to estimate rainfall magnitude at unmeasured locations. This method can provide a realistic estimate of uncertainty for each predicted location. Taking river anisotropy into account, Merwade et al. (2006) proposed an elliptical IDW with an elliptical distance measure to interpolate river channel bathymetry. Results indicated that it provides a simple and computationally faster alternative to complex kriging methods. Some scholars attempted to capture the optimal power parameter and number of neighbors of IDW. For example, Chang et al. (2006) developed a variable-order distance IDW based on the generic algorithm to minimize the difference between estimated and measured precipitation data. Lu and Wong (2008) presented an adaptive IDW, where the power parameter is allowed to vary according to the spatial pattern of the sampled points in the neighborhood. Babak and Deutsch (2009) employed a statistical approach to find the optimal power parameter and number of neighbor points. de Mesnard (2013) argued that, from elementary laws of physics, the optimal power of distance depends on the form of pollution encountered, such as radiant pollution (including radioactivity and sound), air pollution (plumes, puffs, and motionless clouds by using the classical Gaussian model), and polluted rivers. Some researchers pointed out that there are many spatially distributed data sets that are improperly represented by Euclidean distances and require specified distance measures which can accurately represent their complex geographic connectivity. For example, based on the fact that spatial distribution of events observed on a network can be analyzed more accurately with network-based methods, Shiode and Shiode (2011) developed a network-based IDW to help more accurately predict unknown spatial values along networks. Greenberg et al. (2011) employed the cumulative, rasterbased least-cost-distance to define the distance between two locations and found that this new distance-based-IDW yields more realistic interpolation of water temperature. Zhou and Sha (2013) introduced three similarity measures for IDW interpolation, namely, spatial distance measure (SDM), nonspatial attribute similarity measure (NSAM), and their hybrid (SDM+NSASM). Simulation results showed that NSASM and SDM+NSASM presented a better similarity of soil properties between soil points when compared with traditional SDM. Compared with the classical IDW, almost all variants have higher interpolation accuracy for attribute estimation.

Methodologically, IDW is essentially a kind of NadarayaWatson kernel smoother with an inverse distance kernel. It is well known that this smoother always leads to less accurate function estimates than the local linear approach and cannot even estimate a linear surface without bias (Cleveland et al. 1988). Moreover, it suffers from the so-called boundary effect (Cai 2001; Hastie and Loader 1993). In other words, bias of the function estimates is larger at the boundary of the exploratory variable space than in the interior. This is due to the serious asymmetry of sample points around the interpolation location in the kernel neighborhood, when the location is close enough to the boundary.

Recently, the local polynomial modeling has been received much attention in statistics because of its good features including good asymptotic properties of bias and variance of the estimates, good behavior in terms of minimax efficiency, and the ability of automatically correcting the boundary effect (Fan and Gijbels 1996). Wang et al. (2008) empirically proved that the local polynomial modeling obviously reduced the boundary bias of the coefficient estimation of geographically weighted regression (GWR). Therefore, in this paper, the classical IDW is generalized using the local polynomial modeling to avoid the boundary effect and improve its interpolation accuracy.

\section{Generalization of IDW}

IDW is a type of deterministic method for spatial interpolation with a known scattered set of points. The assigned values to unknown points are calculated with a distance-decay weighted average of the values available at the known points. Suppose that a surface is a graph of a function, $z=m(\boldsymbol{x})$, the value of $m(\boldsymbol{x})$ at location $\boldsymbol{x}_{0}$ is estimated by IDW as follows,

$m\left(\boldsymbol{x}_{0}\right)=\sum_{i=1}^{n} w_{i 0} f\left(\boldsymbol{x}_{i}\right) / \sum_{i=1}^{n} w_{i 0}$ 
where $f\left(\boldsymbol{x}_{i}\right)$ represents the value of the $i$ th neighbor sample point around the location $\boldsymbol{x}_{0} ; n$ is the number of neighbor sample points used for spatial interpolation; $w_{i 0}$ is the weight of $f\left(\boldsymbol{x}_{i}\right)$ expressed as,

$w_{i 0}=d_{i 0}^{-p}$

where $d_{i 0}$ is the distance between the point $\boldsymbol{x}_{0}$ and the $i$ th neighbor point; $p$ is the power parameter determining the significance of the sample points upon the interpolated value. If the distance between the sampled location and the interpolated location increases, the weight that the sampled point has on the interpolation decreases (Burrough and McDonnell 1998). So a higher power results in less influence from distant points. It can be shown that estimating $m\left(\boldsymbol{x}_{0}\right)$ with Eq. 1 is equivalent to solving the following optimization problem,

$$
\begin{aligned}
& \arg \min _{m\left(\boldsymbol{x}_{0}\right)} \sum_{i=1}^{n} w_{i 0}\left(f\left(\boldsymbol{x}_{i}\right)-m\left(\boldsymbol{x}_{0}\right)\right)^{2} \\
& =\arg \min _{m\left(\boldsymbol{x}_{0}\right)}\left(\boldsymbol{f}-\boldsymbol{I} m\left(\boldsymbol{x}_{0}\right)\right)^{T} \boldsymbol{W}\left(\boldsymbol{f}-\boldsymbol{I} m\left(\boldsymbol{x}_{0}\right)\right)
\end{aligned}
$$

where $\boldsymbol{f}=\left[\begin{array}{lll}f\left(\boldsymbol{x}_{1}\right) & f\left(\boldsymbol{x}_{2}\right) & \cdots\end{array}\left(\boldsymbol{x}_{n}\right)\right]^{T} ; \boldsymbol{I}$ is a unit matrix, $\mathrm{n}$ a $\mathrm{m} \mathrm{e} 1 \mathrm{y}, \quad \boldsymbol{I}=\left[\begin{array}{llll}1 & 1 & \cdots & 1\end{array}\right]^{T} \quad ; \quad \boldsymbol{W}=\operatorname{diag}$ $\left(\begin{array}{llll}w_{10} & w_{20} & \cdots & w_{n 0}\end{array}\right)$.

The equivalence between Eqs. 1 and 3 can be proved as follows. Letting the first derivative of Eq. 3 with respect to $m\left(\boldsymbol{x}_{0}\right)$ equal to zero, we can obtain,

$\boldsymbol{I}^{T} \boldsymbol{W}\left(\boldsymbol{I} m\left(\boldsymbol{x}_{0}\right)-\boldsymbol{f}\right)=0$

or,

$$
\boldsymbol{I}^{T} \boldsymbol{W} \boldsymbol{I} m\left(\boldsymbol{x}_{0}\right)=\boldsymbol{I}^{T} \boldsymbol{W} \boldsymbol{f}
$$

so,

$$
\begin{aligned}
m\left(\boldsymbol{x}_{0}\right) & =\frac{\boldsymbol{I}^{T} \boldsymbol{W} \boldsymbol{f}}{\boldsymbol{I}^{T} \boldsymbol{W} \boldsymbol{I}} f\left(\boldsymbol{x}_{i}\right) / \sum_{i=1}^{n} w_{i 0} \\
& =\sum_{i=1}^{n} w_{i 0}
\end{aligned}
$$

Equation 3 implicitly indicates that the function values of $m(\boldsymbol{x})$ at $\boldsymbol{x}_{i}(i=1,2, \cdots, n)$ are locally taken to be the same constant in order to estimate $m\left(x_{0}\right)$ using the weighted leastsquares procedure. This idea is similar to that of GWR (Fotheringham et al. 2002). Specifically, the regression coefficients at a focal point are assumed to be locally constant and computed via least-squares method, coupled with a distance-decay kernel specifying the weights. In essence, the classical IDW is only one kind of the Nadaraya-Watson estimators with an inverse distance kernel, which suffer from various shortcomings (Cai 2001).

In local polynomial modeling, the regression function is locally expanded as an $r$-order polynomial of the explanatory variables by the Taylor expansion, and the weighted leastsquares method is used to locally estimate the regression function and its derivatives at each given point in the space of the explanatory variables. Motivated by this methodology, we can locally expand the function $m(\boldsymbol{x})$ at $\boldsymbol{x}_{0}$ to accurately estimate $m\left(x_{0}\right)$ for $\boldsymbol{x}$ in a neighborhood of $\boldsymbol{x}_{0}$.

Assuming that the function $m(\boldsymbol{x})$ is locally sufficient smooth, it can be expanded at a given point $\boldsymbol{x}_{0}$ by the Taylor expansion as follows,

$$
\begin{aligned}
& m(\boldsymbol{x})=m\left(\boldsymbol{x}_{0}\right)+m^{\prime}\left(\boldsymbol{x}_{0}\right)\left(\boldsymbol{x}-\boldsymbol{x}_{0}\right)+\frac{m^{\prime \prime}\left(\boldsymbol{x}_{0}\right)}{2 !}\left(\boldsymbol{x}-\boldsymbol{x}_{0}\right)^{2}+\cdots \\
& =\beta_{0}+\boldsymbol{\beta}_{1}\left(\boldsymbol{x}-\boldsymbol{x}_{0}\right)+\boldsymbol{\beta}_{2}\left(\boldsymbol{x}-\boldsymbol{x}_{0}\right)^{2}+\cdots
\end{aligned}
$$

where $\boldsymbol{\beta}_{i}=\frac{m^{(i)}\left(\boldsymbol{x}_{0}\right)}{i !}$.

This polynomial is locally fitted by a weighted leastsquares problem as follows,

$$
\begin{aligned}
\arg & \min _{\beta} \sum_{i=1}^{n} w_{0 i}\left(f\left(\boldsymbol{x}_{i}\right)-\beta_{0}-\boldsymbol{\beta}_{1}\left(\boldsymbol{x}_{i}-\boldsymbol{x}_{0}\right)-\boldsymbol{\beta}_{2}\left(\boldsymbol{x}_{i}-\boldsymbol{x}_{0}\right)^{2}-\cdots\right)^{2} \\
= & \arg \min _{\boldsymbol{\beta}}(\boldsymbol{f}-\mathrm{B} \boldsymbol{\beta})^{T} \boldsymbol{W}(\boldsymbol{f}-\mathrm{B} \boldsymbol{\beta})
\end{aligned}
$$

where $\boldsymbol{\beta}=\left[\begin{array}{llll}\beta_{0} & \boldsymbol{\beta}_{1} & \boldsymbol{\beta}_{2} & \cdots\end{array}\right]^{T} ; \boldsymbol{B}$

$$
=\left[\begin{array}{cccc}
1 & \left(\boldsymbol{x}_{1}-\boldsymbol{x}_{0}\right) & \left(\boldsymbol{x}_{1}-\boldsymbol{x}_{0}\right)^{2} & \ldots \\
1 & \left(\boldsymbol{x}_{2}-\boldsymbol{x}_{0}\right) & \left(\boldsymbol{x}_{2}-\boldsymbol{x}_{0}\right)^{2} & \ldots \\
\vdots & \vdots & \vdots & \vdots \\
1 & \left(\boldsymbol{x}_{n}-\boldsymbol{x}_{0}\right) & \left(\boldsymbol{x}_{2}-\boldsymbol{x}_{0}\right)^{2} & \ldots
\end{array}\right] .
$$

The solution of the weighted least squares problem in Eq. 8 can be expressed as,

$\boldsymbol{\beta}=\left(\boldsymbol{B}^{T} \boldsymbol{W} \boldsymbol{B}\right)^{-1} \boldsymbol{B}^{T} \boldsymbol{W} \boldsymbol{f}$

Hence, the estimated value at the location $x_{0}$ is $\beta_{0}$. Namely, $m\left(\boldsymbol{x}_{0}\right)=\beta_{0}$.

Comparing Eq. 3 with Eq. 8 , we can find that Eq. 8 is a generalization of Eq. 3. Equation 3 is only the zeroth-order 
case of Eq. 8 with $\boldsymbol{B}$ being a unit vector. Hence, the method of IDW can be generalized to any arbitrary order by determining the number of terms retained in the Taylor expansion. In this paper, only the first- and the second-orders IDWs were tested.

\section{Real-world examples}

Surface modeling of rainfall fields

Spatially distributed rainfall field is one of necessary inputs to many environmental and hydrological models. However, rainfall records are often incomplete because of missing rainfall data in the measured period, or insufficient rainfall stations in the study region. To resolve these problems, absent rainfall data are always estimated through spatial interpolation techniques.

In our test, 750 rain gauges released by the national meteorological network of China were obtained. The Albers projection was adopted to transform geographical coordinates into rectangular Cartesian coordinates. The Albers projection (named after Heinrich C. Albers) is a conic and equal area map projection that uses two standard parallels. Although scale and shape are not preserved, distortion is minimal between the standard parallels. The corresponding elevations of the 750 rain gauges were also obtained from the 1-km Shuttle Radar Topography Mission (SRTM) digital elevation model (DEM) (download from http://westdc.westgis.ac.cn). After the preprocessing, each rain gauge has the attributes of the $(x, y)$ coordinates, elevation, and mean annual rainfall. Considering the completeness of rainfall records with at least 30 year at each station during the period from 1951 to 2010, only 722 stations (Fig. 1) were selected. These points were used for surface modelling of annual mean rainfall field for the period from 1951 to 2010 by the classical IDW and the generalized IDWs, respectively. All the IDW methods obtained their optimal power parameter and number of neighbor sample points with the leave-one-out cross-validation (LOOCV). In principle, LOOCV involves using a single observation from the original sample as the validation data and the remaining observations as the training data. This is repeated until each observation in the sample is used once as the validation data. In LOOCV, the power parameters varied from 1 to 5 and the number of neighbor sample points from 3 to 70 . This wide value range aims to capture the optimal parameters of the three versions of IDW and give them a reasonable and impartial comparison.

Considering the impact of spatial coordinates and elevation on rainfall, the regression function in the generalized IDW was locally expanded as a linear function of spatial coordinates and elevation in this test. The performances of the IDWs with their optimal parameters were quantitatively compared in terms of root mean square error (RMSE) and mean error (ME). RMSE and ME are respectively expressed as,

RMSE $=\sqrt{\frac{\sum_{i=1}^{n}\left(z_{i}-\widehat{z}_{i}\right)^{2}}{n}}$

$\mathrm{ME}=\frac{\sum_{i=1}^{n}\left(z_{i}-\widehat{z}_{i}\right)}{n}$

where $z_{i}$ and $\hat{z}_{i}$ are the $i$ th observed and estimated values, respectively; $n$ is the number of validation points.

Results (Table 1) indicate that the classical IDW and the second-order IDW have the same optimal parameters. Namely, the power parameter is 1 , and the number of neighbor sample points is 50 . Yet, the first-order IDW has the power parameter of 2 and 9 neighbor points. In terms of RMSE, the two generalized IDWs are clearly more accurate than the classical one. This is expected as the two generalized methods use the superior statistical mathematical property of minimum error variance and consider trend in the dataset. However, in terms of ME, the secondorder IDW has the poorest result, which is followed by the classical IDW and the first-order IDW. This shows that the second-order IDW is more biased than the other IDWs. In a word, the second-order IDW might be a good alternative to surface modeling of rainfall field in terms of variance, and the first-order IDW in terms of bias.

Figure 2 shows the relationship between the observed and estimated rainfalls. The results indicate that the classical IDW has the poorest results, as there are many overestimated and underestimated points, which are relatively far from the straight line of $y=x$. Compared with the classical IDW, the two generalized IDWs have obviously better results when the rainfalls are lower than $1,000 \mathrm{~mm}$. In terms of $R^{2}$, the secondorder IDW is slightly more accurate than the first-order IDW and the classical IDW.

Mean annual rainfall maps (Fig. 3) show that there are many 'bull eyes' around the rainfall stations in the classical IDW, which makes the map very coarse. Relatively, the firstand the second-order IDWs produce much smoother maps than the classical IDW, and the 'bull eyes' are less obvious. The difference between the first- and the second-order IDWs is slight, which is difficult to recognize from the maps. However, the minimum and the maximum rainfalls of the second-order IDW are slightly smaller than those of the other IDWs, indicating that the second-order IDW has the obvious effect of extrapolation. This result is expected as the higher- 


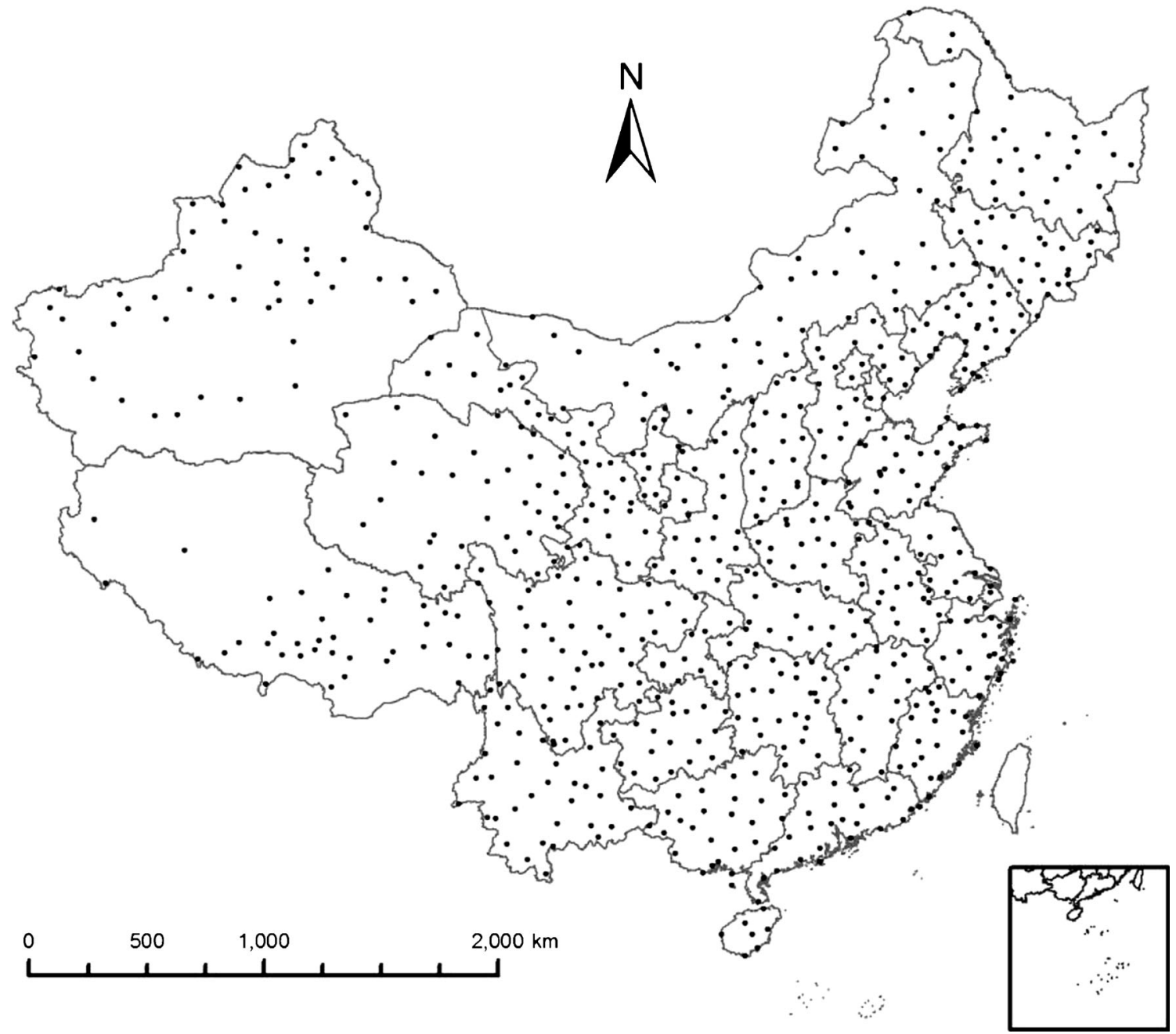

Fig. 1 Spatial distribution of rainfall station network in China

order IDWs allow for the coefficients of the weighted sum in Eq. 9 to have negative values. Yet, the classical IDW always have a positive weight (Eq. 3).

\section{Surface modeling of DEMs}

A DEM is the digital cartographic representation of the elevation of the land at regularly spaced intervals in $x$ and $y$ directions, with $z$-values referenced to a common

Table 1 Accuracy comparison between the classical IDW and the generalized IDWs for surface modeling of rainfall fields

\begin{tabular}{lllll}
\hline Method & $\begin{array}{l}\text { Power } \\
\text { parameter }\end{array}$ & $\begin{array}{l}\text { Number of } \\
\text { neighbors }\end{array}$ & $\begin{array}{l}\text { RMSE } \\
(\mathrm{mm})\end{array}$ & $\begin{array}{l}\text { ME } \\
(\mathrm{mm})\end{array}$ \\
\hline Classical IDW & 1 & 50 & 160 & -8 \\
First-order IDW & 2 & 9 & 147 & 6 \\
Second-order IDW & 1 & 50 & 145 & 10 \\
\hline
\end{tabular}

vertical datum (Burrough and McDonnell 1998; Fisher and Tate 2006). DEMs have been widely adopted in various applications, such as geomorphological and hydrogeological modeling (El Bastawesy 2014; Wakode et al. 2013), viewshed analysis (Fisher 1992), and the correction of digital satellite Imagery (Van Niel et al. 2008). DEMs can be constructed based on an interpolation method with a series of sample points.

In this research, the study site is located in Jinan city, Shandong province, China. Its elevations vary from 109 to $358 \mathrm{~m}$ with the standard deviation of $50 \mathrm{~m}$ and mean of $173.5 \mathrm{~m}$. The 2,742 sample points with the vertical and horizontal accuracies of 10 and $5 \mathrm{~cm}$ were randomly collected by a total station instrument. The $10-\mathrm{m}$ DEMs were respectively constructed by the classical IDW, the generalized IDWs with the first- and the second-order, and ordinary kriging (OK). OK was performed in the GS+geostatistics software (Robertson 2008). GS+provides all geostatistics components, from semivariance analysis through kriging and mapping, in a 
(a)

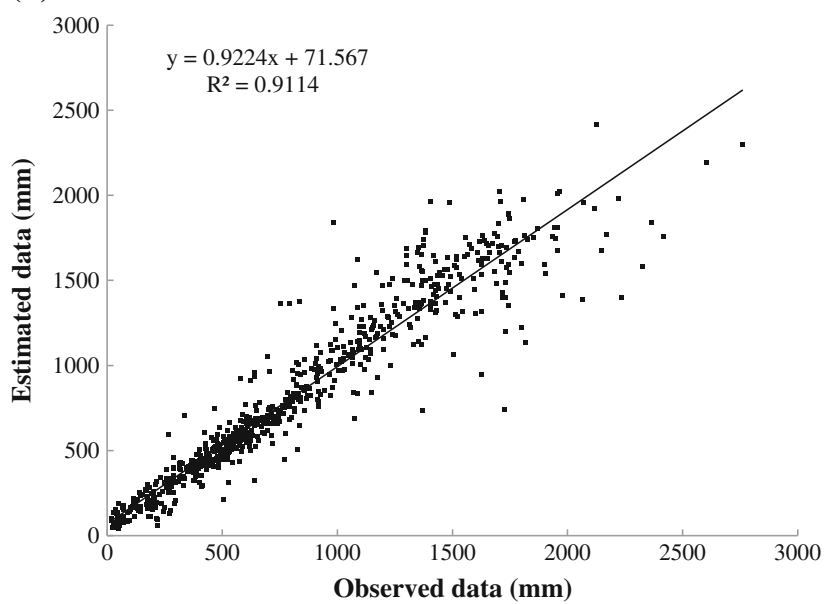

(b)

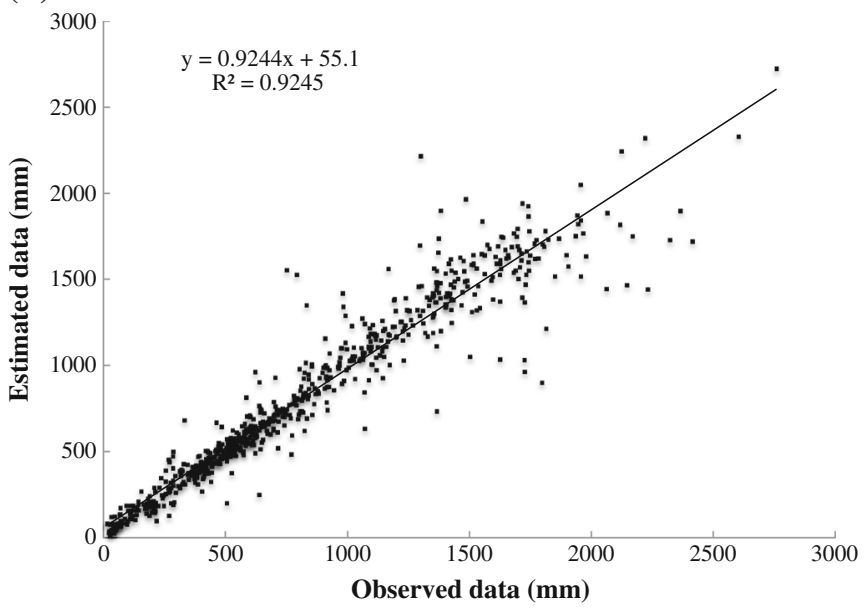

(c)

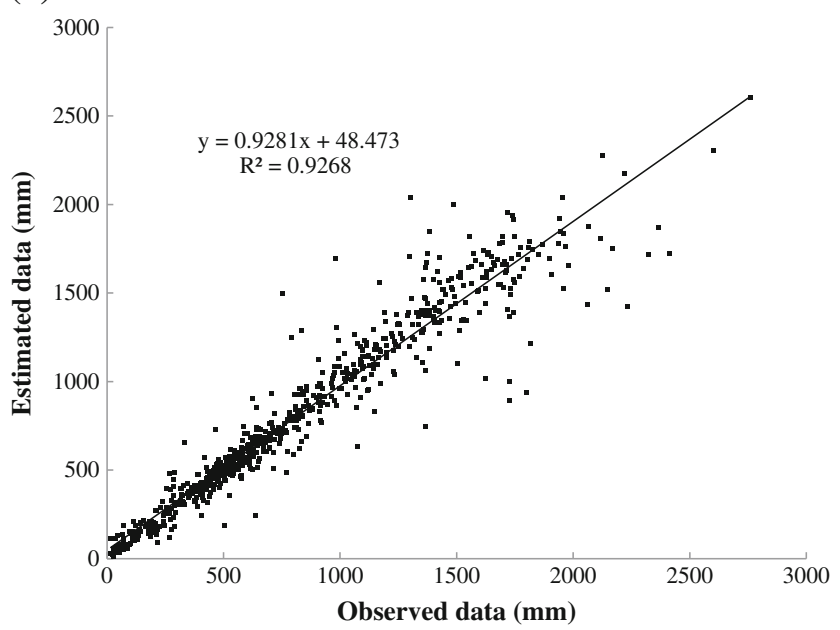

Fig. 2 Accuracy comparisons between the estimated and observed mean annual rainfalls of a the classical IDW, $\mathbf{b}$ the first-order IDW, and $\mathbf{c}$ the secondorder IDW

single integrated software program widely praised for its flexibility and friendly interface. In order to test the interpolation accuracy, 503 check points (Fig. 4) were sampled by RKT GPS with a vertical accuracy of $2 \mathrm{~cm}$ and horizontal accuracy of $1 \mathrm{~cm}$, respectively.

For OK, the semivariogram and its parameters were shown in Fig. 5. We found that it is an exponential model with the nugget variance $\left(C_{0}\right)$ of $1.0 \mathrm{~m}^{2}$, sill $\left(C_{0}+C\right)$ of $2,661 \mathrm{~m}^{2}$, residual sum of squares of $76,814 \mathrm{~m}^{2}$, range $\left(A_{0}\right)$ of $547 \mathrm{~m}$, and fitting $R^{2}$ of 0.991 , respectively. The three versions of IDW have the same optimal power parameter of 1 , whereas the number of neighbor points is different (Table 2). The first-order IDW has the minimum number of 6 , and the second-order IDW has the maximum number of 22 . Table 2 demonstrates that the classical IDW has the poorest performance with the RMSE and ME of $3.76 \mathrm{~m}$ and $-0.16 \mathrm{~m}$, respectively. The other three methods are much more accurate than the classical IDW, regardless of RMSE and ME. It should be noted that, although the semivariogram of OK was good fitted by the exponential model, the second-order IDW performs slightly better than OK in terms of RMSE. This result may be inconsistent with the common sense that, when the spatial structure of data points was accurately captured, OK is always more accurate than IDW.

The computing time of the three versions of IDW was also shown in Table 2. Considering that the semivariogram of $\mathrm{OK}$ must be manually obtained and its cost is difficult to count, its time was not listed. We can see that the second-order IDW has the most time cost, which is closely followed by the first-order IDW and the classical IDW. This result can be expected as for the second-order IDW, the order of $\boldsymbol{B}^{T} \boldsymbol{W} \boldsymbol{B}$ in Eq. 9 is 6 while, for the first-order IDW, the order is 3 . Furthermore, the classical IDW need not solve the 
(a)

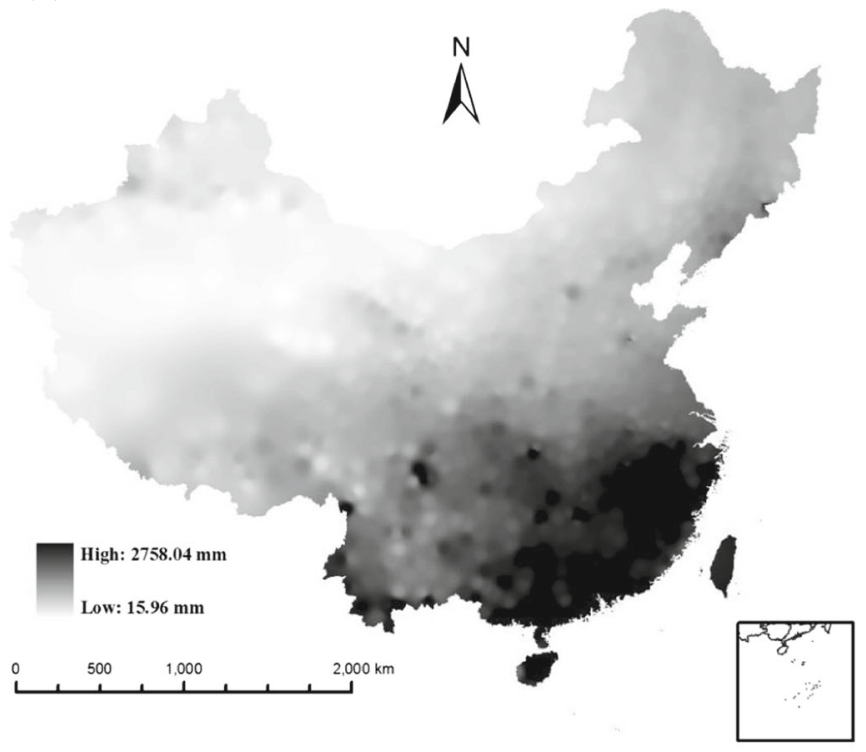

(b)

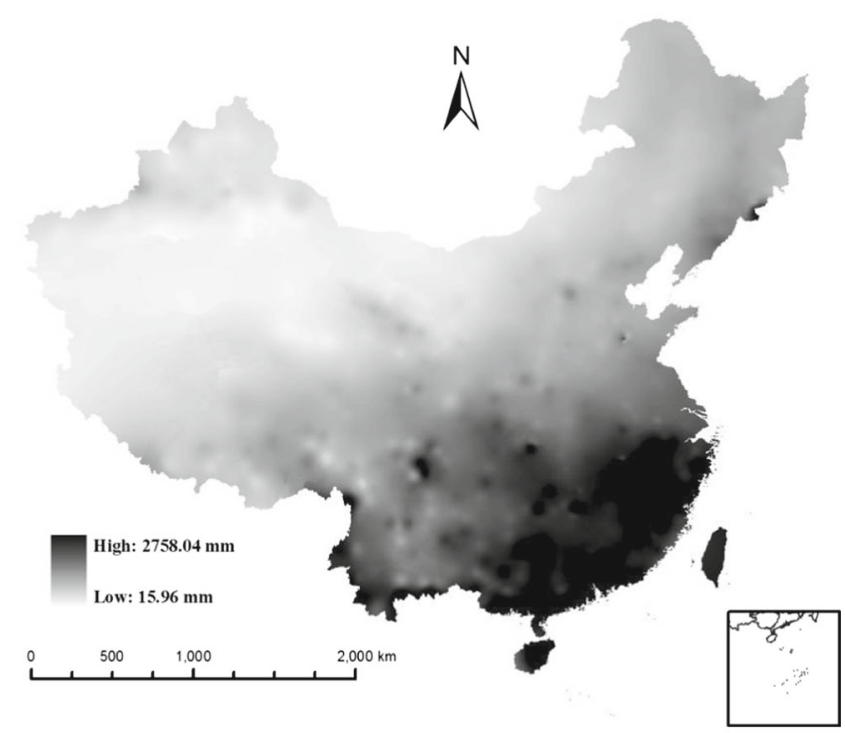

(c)

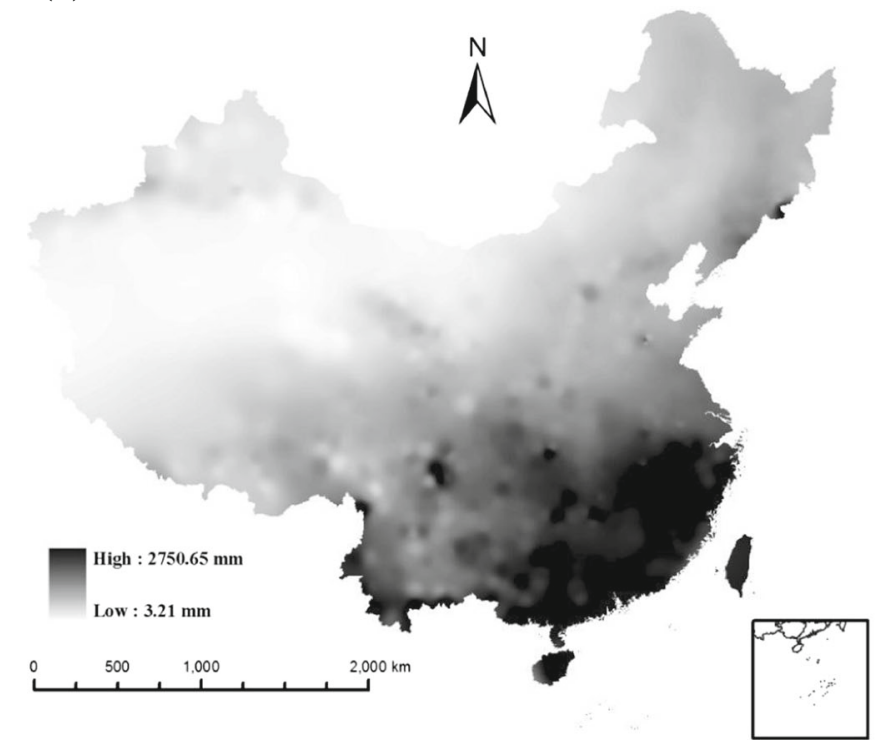

Fig. 3 Mean annual rainfall maps of a the classical IDW, $\mathbf{b}$ the first-order IDW, and $\mathbf{c}$ the second-order IDW

system of linear equations like Eq. 9. However, the time difference among the three IDWs is minor. This is due to the fact that IDWs always cost much more time to find the neighbor sample points than to perform interpolations.

The shaded relief maps of the four interpolation methods (Fig. 6) show that the classical IDW obtained the coarsest surface, especially in the left and right boundary areas. The map of the first-order IDW is much smoother than that of the classical IDW. However, there are still many isolated artificial pinks with a random distribution. Compared with other maps, those of the second-order IDW and kriging have a good appearance. Nevertheless, kriging seems to have an obvious smoothing effect, which causes a peak-cutting and valley-filling problem.

\section{Discussion and conclusions}

Discussion

Specifically, taking the spatial coordinates $(x, y)$ and elevation $(e)$ as the independent variables, Eq. 8 with 
Fig. 4 Check points and shaded relief map of the study site
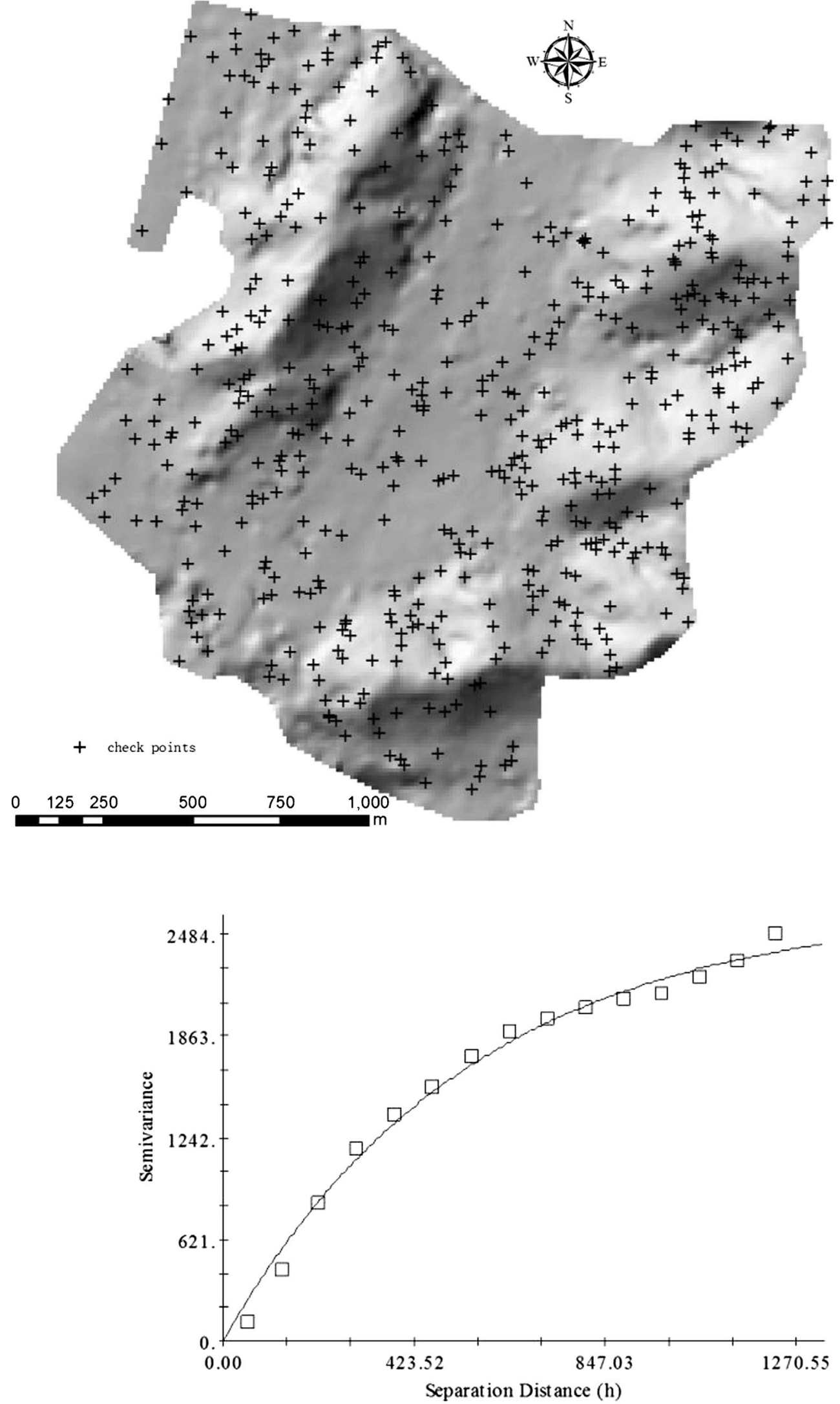

Exponential model $(\mathrm{Co}=1.00000 ; \mathrm{Co}+\mathrm{C}=2661.00000 ; \mathrm{Ao}=547.00 ; \mathrm{r} 2=0.991 ;$ RSS $=76814$.) 
Table 2 Accuracy comparison between IDWs and OK for surface modeling of DEMs

\begin{tabular}{lllllc}
\hline Method & $\begin{array}{l}\text { Power } \\
\text { parameter }\end{array}$ & $\begin{array}{l}\text { Number of } \\
\text { neighbors }\end{array}$ & RMSE (m) & ME (m) & $\begin{array}{l}\text { Computing } \\
\text { time (s) }\end{array}$ \\
\hline Classical IDW & 1 & 7 & 3.76 & -0.16 & 201.3 \\
First-order IDW & 1 & 6 & 2.94 & -0.03 & 202.6 \\
Second-order IDW & 1 & 22 & 2.35 & 0.01 & 209.4 \\
OK & - & 16 & 2.37 & -0.01 & - \\
\hline
\end{tabular}

(a)

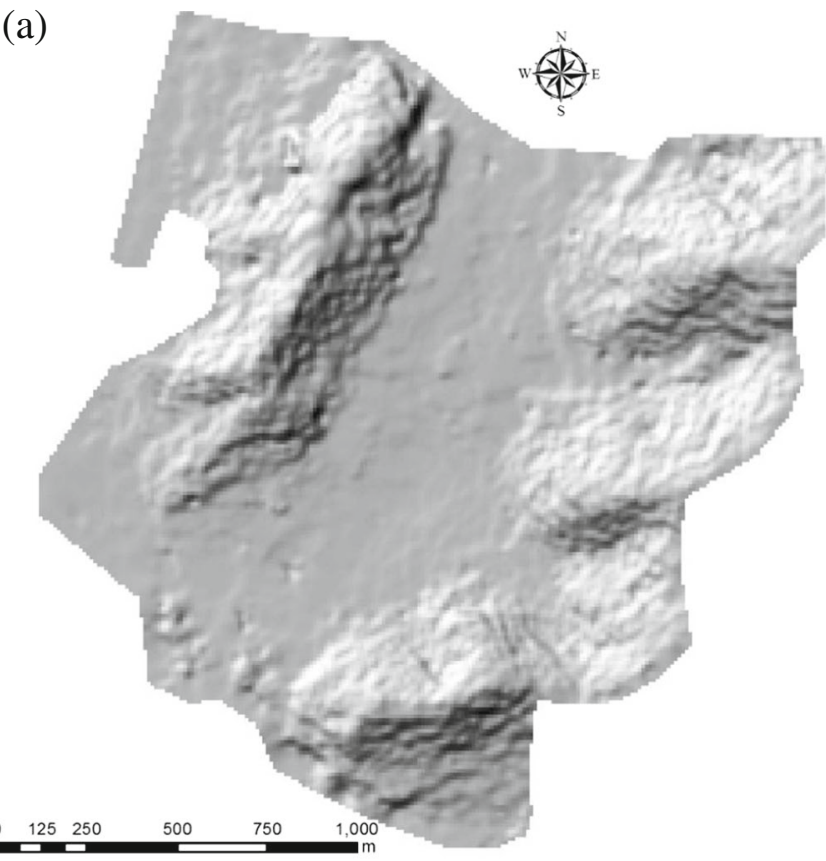

(c)

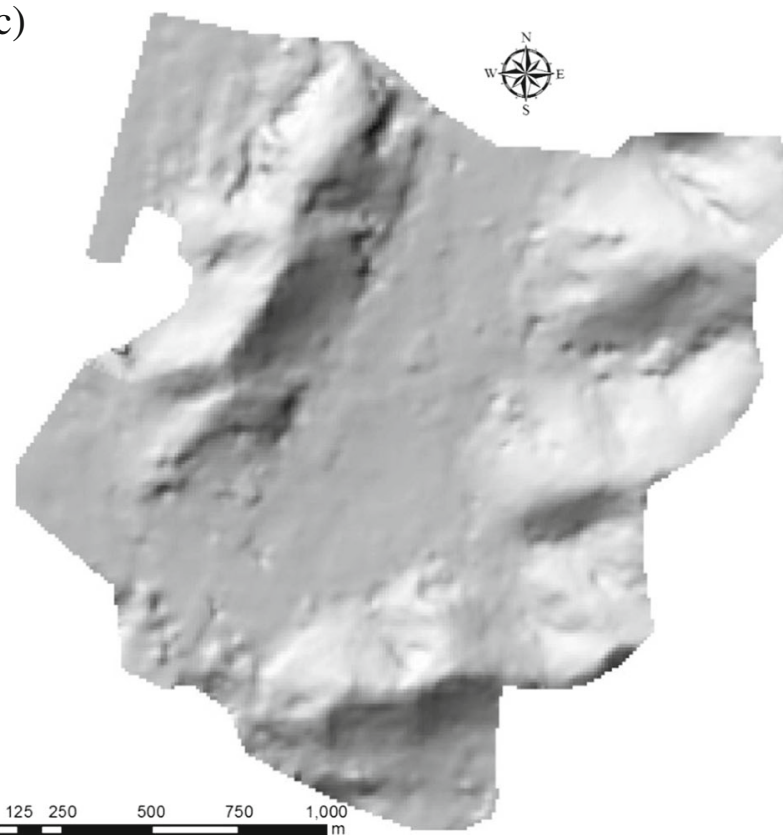

(b)

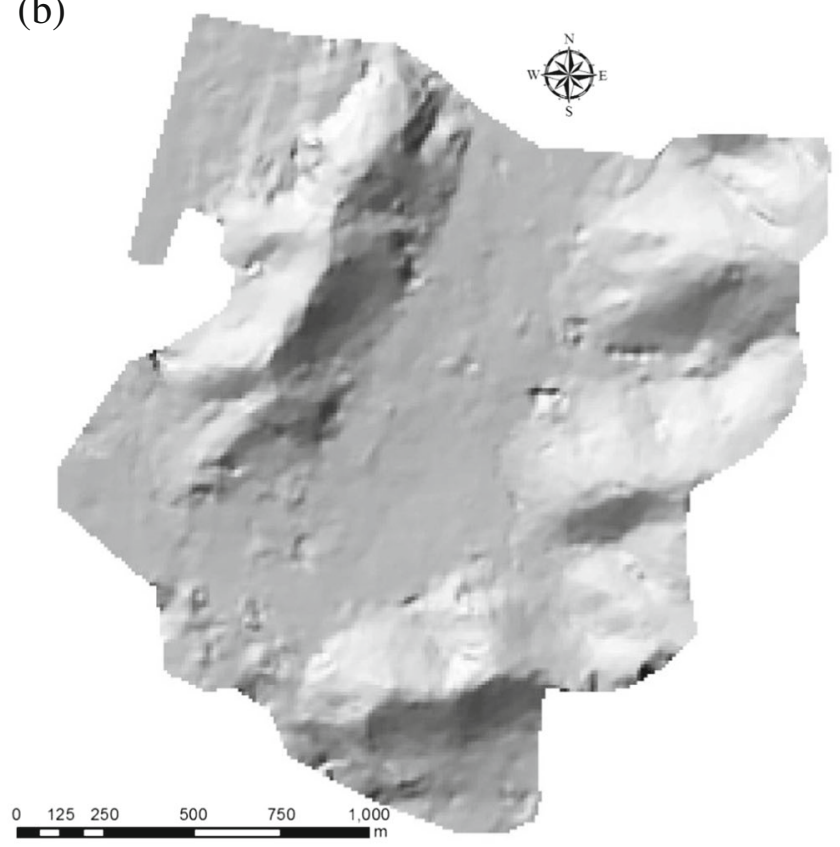

(d)

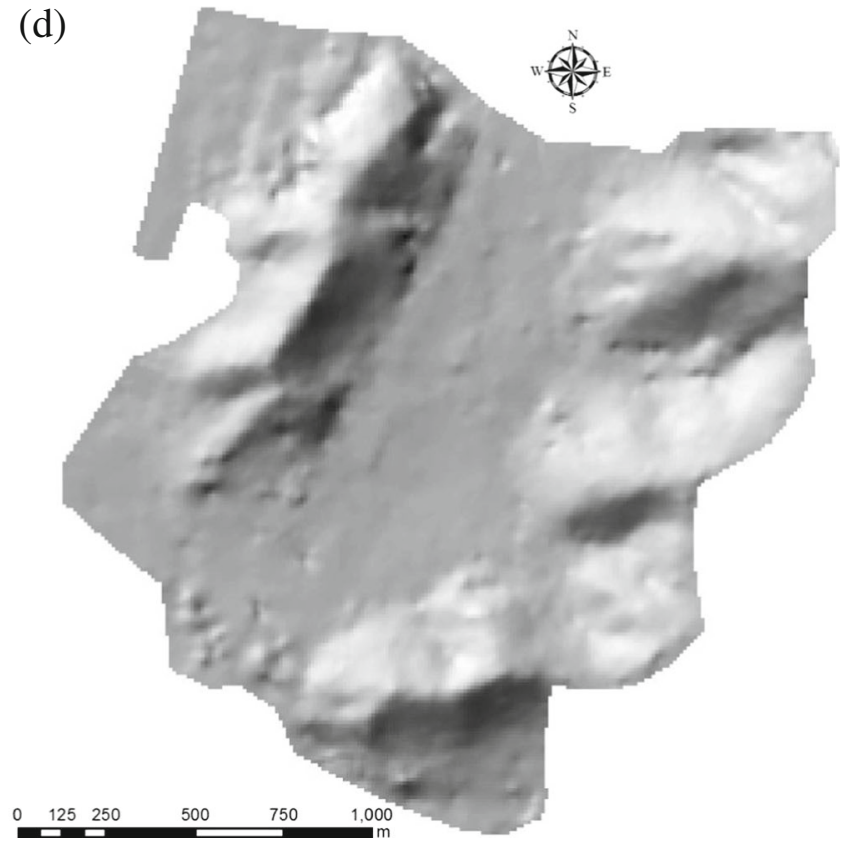

Fig. 6 Shaded relief maps of a the classical IDW, $\mathbf{b}$ the first-order IDW, $\mathbf{c}$ the second-order IDW, and $\mathbf{d}$ OK 
the first-order expansion can be transformed into the following form,

$$
\begin{aligned}
& \arg \min _{\beta_{0}} \sum_{i=1}^{n} w_{i 0}\left(f_{i}-\beta_{0}-\beta_{1}\left(x_{i}-x_{0}\right)-\beta_{2}\left(y_{i}-y_{0}\right)-\beta_{3}\left(e_{i}-e_{0}\right)\right)^{2} \\
& =\arg \min _{\beta_{0}} \sum_{i=1}^{n} w_{i 0}\left(\beta_{0}-\left(f_{i}+\beta_{1}\left(x_{0}-x_{i}\right)+\beta_{2}\left(y_{0}-y_{i}\right)+\beta_{3}\left(e_{0}-e_{i}\right)\right)\right)^{2} \\
& =\arg \min _{\beta_{0}} \sum_{i=1}^{n} w_{i 0}\left(\beta_{0}-l_{i}\right)^{2}
\end{aligned}
$$

where $l_{i}=f_{i}+\beta_{1}\left(x_{0}-x_{i}\right)+\beta_{2}\left(y_{0}-y_{i}\right)+\beta_{3}\left(e_{0}-e_{i}\right)$.

In terms of the weighted least squares method, we can obtain,

$$
\begin{aligned}
\beta_{0}= & \sum_{i=1}^{n}\left(w_{i 0} l_{i}\right) / \sum_{i=1}^{n} w_{i 0} \\
= & \sum_{i=1}^{n}\left(w_{i 0}\left(f_{i}+\beta_{1}\left(x_{0}-x_{i}\right)+\beta_{2}\left(y_{0}-y_{i}\right)+\beta_{3}\left(e_{0}-e_{i}\right)\right)\right) \\
& / \sum_{i=1}^{n} w_{i 0}
\end{aligned}
$$

It should be noted that kriging and IDW are completely different interpolation methods, theoretically. The purpose of this paper is not to replace kriging by the generalized IDW but to improve the performance of the classical IDW for practical applications. However, when the spatial structure of sample points is low or difficult to capture, the generalized IDWs might take place of kriging for surface modeling.

\section{Conclusions}

In this paper, we show that the classical IDW is a zeroth-order kernel regression method with an inverse distance kernel. Therefore, it is generalized based on the local polynomial fitting and then estimated by the weighted least-squares procedure. Surface modeling of rainfall fields indicates that the first- and the second-order IDWs have much better performance than the classical IDW in terms of RMSE and $R^{2}$. The example of DEM construction based on randomly distributed sample points shows that the generalized IDWs have better RMSE and ME than the classical IDW. Furthermore, the second-order IDW performs slightly better than the ordinary kriging in terms of RMSE. In conclusion, the generalized IDWs seem to be an alternative to the classical IDW for surface modeling.

Acknowledgments The 1-km SRTM data used in this paper were provided by Environmental and Ecological Science Data Center for West China, National Natural Science Foundation of China (http://westdc. westgis.ac.cn). This work is supported by National Natural Science Foundation of China (Grant No. 41101433, 41371367), by Young and Middle-Aged Scientists Research Awards Fund of Shangdong Province (Grant No. BS2012HZ010), by Qingdao Science and Technology Program of Basic Research Project (Grant No. 13-1-4-239-jch), by the Key Laboratory of Marine Surveying and Mapping in Universities of Shandong (Shandong University of Science and Technology) (Grant No. 2013B03), by SDUST Research Fund, and by Joint Innovative Center for Safe and Effective Mining Technology and Equipment of Coal Resources.

\section{References}

Babak O, Deutsch C (2009) Statistical approach to inverse distance interpolation. Stoch Env Res Risk A 23(5):543-553

Boman G, Molz F, Guven O (1995) An evaluation of interpolation methodologies for generating three-dimensional hydraulic property distributions from measured data. Ground Water 33(2):247-258

Brus DJ, Gruijter JJD, Marsman BA, Visschers R, Bregt AK, Breeuwsma A, Bouma J (1996) The performance of spatial interpolation methods and choropleth maps to estimate properties at points: a soil survey case study. Environmetrics 7(1):1-16

Burrough PA, McDonnell RA (1998) Principles of geographical information systems. Oxford University Press, New York, p 220

Cai Z (2001) Weighted Nadaraya-Watson regression estimation. Stat Probabil Lett 51(3):307-318 
Chang C-L, Lo S-L, Yu S-L (2006) The parameter optimization in the inverse distance method by genetic algorithm for estimating precipitation. Environ Monit Assess 117(1-3):145-155

Chen CF, Yue TX (2010) A method of DEM construction and related error analysis. Comput Geosci-uk 36(6):717-725

Cleveland WS, Devlin SJ, Grosse E (1988) Regression by local fitting: methods, properties, and computational algorithms. J Econ 37(1): $87-114$

de Mesnard L (2013) Pollution models and inverse distance weighting: some critical remarks. Comput Geosci-uk 52:459-469

Delbari M (2014) Accounting for exhaustive secondary data into the mapping of water table elevation. Arab J Geosci 7(10):4221-4233

Efron B, Hastie T, Johnstone I, Tibshirani R (2004) Least angle regression. Ann Stat 32(2):407-499

El Bastawesy M (2014) The geomorphological and hydrogeological evidences for a Holocene deluge in Arabia. Arab J Geosci 1-10

Falivene O, Cabrera L, Tolosana-Delgado R, Sáez A (2010) Interpolation algorithm ranking using cross-validation and the role of smoothing effect. A coal zone example. Comput Geosci-uk 36(4):512-519

Fan J, Gijbels I (1996) Local polynomial modelling and its applications. CRC Press, London, p 328

Fisher PF (1992) First experiments in viewshed uncertainty: simulating fuzzy viewsheds. Photogramm Eng Remote Sense 58:345

Fisher PF, Tate NJ (2006) Causes and consequences of error in digital elevation models. Prog Phys Geogr 30(4):467-489

Fotheringham A, Brunsdon C, Charlton M (2002) Geographically weighted regression: the analysis of spatially varying relationships. Wiley, Chichester, p 284

Gallichand J, Marcotte D (1993) Mapping clay content for subsurface drainage in the Nile Delta. Geoderma 58(3-4):165-179

Greenberg JA, Rueda C, Hestir EL, Santos MJ, Ustin SL (2011) Least cost distance analysis for spatial interpolation. Comput Geosci-uk 37(2):272-276

Hastie T, Loader C (1993) Local regression: automatic kernel carpentry. Stat Sci 8:120-129

Khashei-Siuki A, Sarbazi M (2013) Evaluation of ANFIS, ANN, and geostatistical models to spatial distribution of groundwater quality (case study: Mashhad plain in Iran). Arab J Geosci 7(10):4175-4190

Kravchenko AN (2003) Influence of spatial structure on accuracy of interpolation methods. Soil Sci Soc Am J 67(5):1564-1571

Lloyd CD (2005) Assessing the effect of integrating elevation data into the estimation of monthly precipitation in Great Britain. J Hydrol 308(1-4):128-150
Lu YG, Wong DW (2008) An adaptive inverse-distance weighting spatial interpolation technique. Comput Geosci-uk 34(9):1044-1055

Merwade VM, Maidment DR, Goff JA (2006) Anisotropic considerations while interpolating river channel bathymetry. J Hydrol 331(3-4):731-741

Moore ID, Grayson RB, Ladson AR (1991) Digital terrain modelling: a review of hydrological, geomorphological, and biological applications. Hydrol Process 5(1):3-30

Nalder IA, Wein RW (1998) Spatial interpolation of climatic Normals: test of a new method in the Canadian boreal forest. Agric For Meteorol 92(4):211-225

Oliver MA, Webster R (1990) Kriging: a method of interpolation for geographical information systems. Int $\mathrm{J}$ Geogr Inf Sci $4(3): 313-332$

Robertson GP (2008) Geostatistics for environmental sciences, GS+ users guide, version 5. Gamma Design Software, Plainwell, p 200

Shahbeik S, Afzal P, Moarefvand P, Qumarsy M (2014) Comparison between ordinary kriging $(\mathrm{OK})$ and inverse distance weighted (IDW) based on estimation error. Case study: Dardevey iron ore deposit, NE Iran. Arab J Geosci 7(9):3693-3704

Shiode N, Shiode S (2011) Street-level spatial interpolation using network-based IDW and ordinary kriging. Trans GIS 15(4): 457-477

Tomczak M (1998) Spatial interpolation and its uncertainty using automated anisotropic inverse distance weighting (IDW)-cross-validation/jackknife approach. J Geogr Inf Decis Anal 2(2):18-30

Van Niel TG, McVicar TR, Li L, Gallant JC, Yang Q (2008) The impact of misregistration on SRTM and DEM image differences. Remote Sens Environ 112(5):2430-2442

Wakode H, Dutta D, Desai VR, Baier K, Azzam R (2013) Morphometric analysis of the upper catchment of Kosi River using GIS techniques. Arab J Geosci 6(2):395-408

Wang N, Mei CL, Yan XD (2008) Local linear estimation of spatially varying coefficient models: an improvement on the geographically weighted regression. Environ Plan A 40:986-1005

Weber D, Englund E (1992) Evaluation and comparison of spatial interpolators. Math Geol 24(4):381-391

Zhou J, Sha Z (2013) A new spatial interpolation approach based on inverse distance weighting: case study from interpolating soil properties. In: Bian F, Xie Y, Cui X, Zeng Y (eds) Geoinformatics in resource management and sustainable ecosystem. Communications in computer and information science. Springer, Berlin, pp 623-631 